\title{
Linear PWM Techniques of Asymmetrical Six-phase Machine With Optimal Current Ripple Performance
}

\author{
Sayan Paul, Kaushik Basu, Senior Member, IEEE
}

\begin{abstract}
Linear modulation techniques (LMTs) of an asymmetrical six-phase machine (ASPM) with two isolated neutral points synthesize the desired voltage vectors by applying at least five switching states. Different choices of applied voltage vectors, sequences in which they are used, distribution of dwelltimes among the redundant switching states give rise to a large number of possible LMTs. It is desirable that these LMTs avoid more than two transitions of a particular inverter leg within a carrier period. Only a subset of existing LMTs of ASPM follows this rule. Through an innovative approach, this paper finds a way to account for all possible infinitely many LMTs that follow the rule of at most two transitions per leg. Another important criterion for the selection of an LMT is its current-ripple performance. Therefore, through numerical optimization, the paper finds optimal LMTs among the above infinite possible LMTs for all reference voltage vectors in the linear range and for the whole feasible range of a machine parameter. This parameter is related to the leakage inductance of the machine and impacts the current ripple performance of ASPM. An optimal hybrid strategy is proposed with these optimal techniques, which outperforms all existing techniques in terms of the current ripple. The theoretical analysis is validated through simulation in Matlab and experiments performed up to $3.5 \mathrm{~kW}$ on a hardware prototype.
\end{abstract}

Index Terms-Multiphase machines, pulse width modulation (PWM), linear PWM technique, asymmetrical six-phase induction motor, six-phase drives, current-ripple.

\section{INTRODUCTION}

$\mathbf{M}$ ULTI-PHASE machines are popular in high-power drives, electric vehicles and railway traction, safetycritical electric aircraft, ship-propulsion etc. due to having advantages of reduced power rating of per-phase powerelectronic drive unit, better fault-tolerance, lesser susceptibility towards space and supply harmonics, [1]-[4]. Asymmetrical six-phase machine (ASPM), one of the most common multiphase machines, has two sets of balanced three-phase $(3 \phi)$ windings with spatial angular difference of $30^{\circ}$ electrical, as shown in Fig. 1.

Vector-space decomposition based modeling of ASPM, as proposed by [5], transforms the variables from original domain to three two-dimensional orthogonal subspaces, namely, $\alpha-\beta$, $z_{1}-z_{2}$ and $o_{1}-o_{2}$. It is shown that electromagnetic energy transfer occurs only in $\alpha-\beta$ plane whereas the remaining two planes don't have coupling between the stator and rotor and hence, are not associated with energy transfer. But, the impedances offered by these two planes are small, so very small excitation in these planes can cause large current and associated copper loss. For two balanced sets of windings with two isolated neutrals, as shown in Fig. $1, o_{1}-o_{2}$ plane can't be excited. Linear modulation techniques (LMTs) of ASPM synthesize the desired average voltages in $\alpha-\beta$ plane and

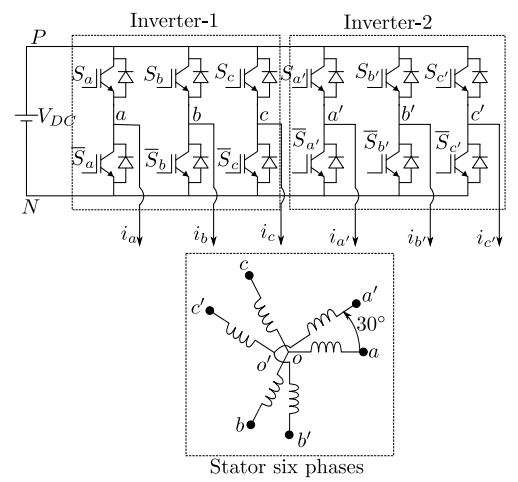

Fig. 1: Six-phase inverter fed ASPM

zero average voltage in $z_{1}-z_{2}$ to avoid the unwanted copper loss. If modulation index $\left(M_{I}\right)$ is defined as the ratio of peak of fundamental line-neutral voltage and DC-bus voltage, the maximum index attainable by LMTs is 0.577 . This paper focusses on these LMTs and their high-frequency currentripple performances.

The proposed space-vector based PWM (SVPWM) technique in [5] shows that LMTs of ASPM need to apply at least five distinct vectors. One such set of five vectors, adopted by [5], [6], is 4 largest active vectors in $\alpha-\beta$ plane, adjacent to the reference voltage vector, $\vec{V}_{r e f}$, and one zero vector. By placing multiple redundant switching states, corresponding to the zero-vector, at different positions in the switching sequences, [6] has proposed continuous and discontinuous linear SVPWM techniques with different current-ripple performances, C6 $\phi$ SVPWM12, D6 $\phi$ SVPWM12-A/B1/B2. [6] also shows that the current ripple of ASPM is not only function of PWM techniques, but it also depends upon machine parameter, $\gamma$, which is defined as the ratio of high-frequency inductances seen by $\alpha-\beta$ and $z_{1}-z_{2}$ subspaces. To discuss the disadvantages of the proposed SVPWM12 techniques of [6], two desirable features of a PWM technique, as given by $\mathbf{R} 1$ and $\mathbf{R 2}$ in the following paragraph, will be discussed first.

R1. Each semiconductor device of the six-phase inverter is allowed to turn-on and tun-off maximum one time over a carrier cycle.

R2. The switching sequence will be mirror symmetric along carrier half-period.

Non-compliance of $\mathbf{R} 1$ results in increase in instantaneous switching frequency of a particular leg for the same average switching frequency, [7], [8]. Carrier based simple implementation is possible if PWM techniques follow rules R1 and R2, [8]. Note, most of the well-known PWM techniques of two-level three-phase inverter, such as SPWM, SVPWM, THIPWMs and DPWMs, follow these rules for carrier-based 
implementation, [9]. None of the PWM techniques, proposed by [6], follows R1. [7] proposed 24 sector based continuous and discontinuous PWM techniques, C $6 \phi$ SVPWM 24 , D6 $\phi$ SVPWM24-B1/B2, where the furthest of the four largest vectors in $\alpha-\beta$ is replaced by one small vector. These techniques follow both the rules. The ripple current performance of D6 $\phi \mathrm{SVPWM} 24-\mathrm{B} 2$ (the best technique in [7]) is worse than that of D6 $\phi$ SVPWM12-B1/B2 techniques for the same average switching frequency at higher $M_{I}$ and higher values of $\gamma$. A modified SVPWM24 technique, C6 $\phi$ SVPWM24-C, is proposed in [8] which also obeys R1 and $\mathbf{R} 2$ but current-ripple performance is not good at higher $M_{I}$ and higher values of $\gamma$.

Another group of LMTs were proposed by [10], [11] where two $3 \phi$ inverters, Inverter- 1 and Inverter- 2 of Fig. 1 , are modulated with the reference voltage vectors $\frac{\vec{V}_{r e f}}{2}$ and $\frac{\vec{V}_{r e f}}{2} e^{-j 30^{\circ}}$, respectively. [12] has shown that this operation is equivalent to synthesizing $\vec{V}_{\text {ref }}$ in $\alpha-\beta$ and zero in $z_{1}-z_{2}$ on average. These techniques are termed as two-inverter based technique (TINV) in [12]. Existing TINV techniques are- a) Sinetriangle PWM (STPWM), [13], where zero common-mode voltage is injected between load-neutral and DC-bus midpoint; b) Double zero-sequence injection PWM (DZIPWM), which is also named as ZS3PWM in [13], where two different zero-sequence signals are used for two $3 \phi$ inverters and the modulation signals of both inverters are compared with same carrier signal; these zero-sequence signals are half of the middle values of the corresponding $3 \phi$ modulation signals, c) ZS6PWM, [13], where a single common-mode signal is used for both Inverter- 1 and Inverter- 2 and this is equal to the half of negative summation of maximum and minimum of $6 \phi$ modulating waves. These three TINV techniques follow rules $\mathbf{R 1}$ and R2. The comparison of current ripple performances as function of $M_{I}$ of these TINV techniques and SVPWM techniques reveals that SVPWM techniques are much better than TINV techniques for the feasible range of $\gamma$, [6], [13], [14].

Note, existing three TINV techniques, three techniques proposed in [7], and C6 $\phi$ SVPWM24-C satisfy the desirable two rules, $\mathbf{R} 1$ and $\mathbf{R 2}$. In fact, there are infinitely many PWM techniques, not just these seven techniques known in the literature, which satisfy these desirable conditions. This infinite possibility arises due to infinite number of choices of distributions of the dwell-times between two redundant zero states of both $3 \phi$ inverters, Inverter- 1 and Inverter- 2 of Fig. 1, in the sequence. It is known from the two-level $3 \phi$ inverter literature that this distribution impacts the current ripple, [9], [15], [16]. In this context, this paper makes following contributions.

1) First attempts to identify the complete set of possible LMTs, which satisfy rules $\mathbf{R} \mathbf{1}$ and $\mathbf{R 2}$, in section II.

2) Then through systematic analysis in section III, it finds the optimal technique that results into minimum current ripple for a given $\vec{V}_{\text {ref }}$ in $\alpha-\beta$, machine parameter, $\gamma$, and given average switching frequency.

3) In section III, it finds all such optimal techniques for all possible $\vec{V}_{\text {ref }}$ in linear range for feasible range of $\gamma$.

4) Finally, it proposes a hybrid LMT in section IV using the
TABLE I: Switching States of Three-phase Inverter

\begin{tabular}{cccc|cccc}
\hline$S_{a}$ & $S_{b}$ & $S_{c}$ & Label & $S_{a}$ & $S_{b}$ & $S_{c}$ & Label \\
\hline Off & Off & Off & 0 & Off & On & On & 4 \\
On & Off & Off & 1 & Off & Off & On & 5 \\
On & On & Off & 2 & On & Off & On & 6 \\
Off & On & Off & 3 & On & On & On & 7 \\
\hline
\end{tabular}

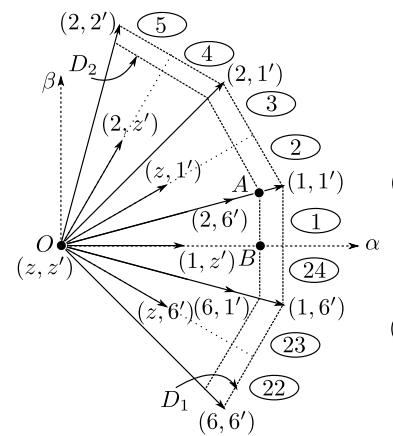

(a) $\alpha-\beta$ plane

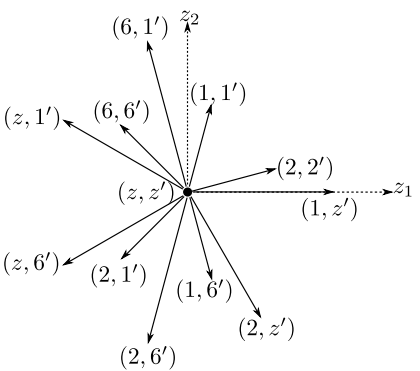

(b) $z_{1}-z_{2}$ plane
Fig. 2: Mapping of 19 states adjacent to sector-1

optimal techniques obtained in previous step. This LMT outperforms all existing LMTs, which follow R1 and R2, in terms of line current THD.

The superiority of the proposed hybrid technique is validated through experiments and simulations in section V. The paper has been concluded in section VI.

\section{Proposed Two-INVERTER BASEd LineAR Modulation TECHNiQues}

Fig. 1 shows a two-level six-phase $(6 \phi)$ inverter, which can be seen as two three-phase $(3 \phi)$ inverters, Inverter- 1 and Inverter-2, respectively, connected across same DC-bus. The pole points of these two $3 \phi$ inverters, $a, b, c$, and $a^{\prime}, b^{\prime}, c^{\prime}$ are directly connected to the terminals of two sets of $3 \phi$ windings of an asymmetrical six-phase machine (ASPM). These two sets of windings are spatially shifted by $30^{\circ}$ electrical and are connected in star with two isolated neutral points $o$ and $o^{\prime}$. This $6 \phi$ inverter has 64 switching states. These states are denoted by an ordered pair, $\left(p, q^{\prime}\right)$, where $p$ and $q$ denote the switching states of Inverter-1 and Inverter-2, respectively, according to the standard $3 \phi$ inverter notation as given in Table I.

The matrix, $T$, as given in (1), was proposed by [5] to model ASPM in three two-dimensional orthogonal subspaces, namely, $\alpha-\beta, z_{1}-z_{2}$ and $o_{1}-o_{2}$. This matrix $T$ is used to map the line-neutral voltages generated by 64 states of $6 \phi$ inverter into the above six orthogonal planes. It is seen that all 64 states generate zero voltages in $o_{1}-o_{2}$ plane and therefore, discussion in $O_{1}-O_{2}$ plane is excluded in the subsequent sections. The voltage vectors generated by 64 switching states in $\alpha-\beta$ and $z_{1}-z_{2}$ subspaces can be referred from [5], [13]. These 64 states generate 24 equivalent sectors in $\alpha-\beta$, [7]. The modulation in one of these 24 sectors in $\alpha-\beta$, which is conventionally called as sector-1 (as shown in Fig. 2a), can be discussed with the help of 19 switching states. Fig. 2 shows the mapping of these 19 switching states in $\alpha-\beta$ and $z_{1}-z_{2}$ planes and they result into 12 distinct vectors in both the subspaces. In Fig. 2, $z$ denotes zero-state and 


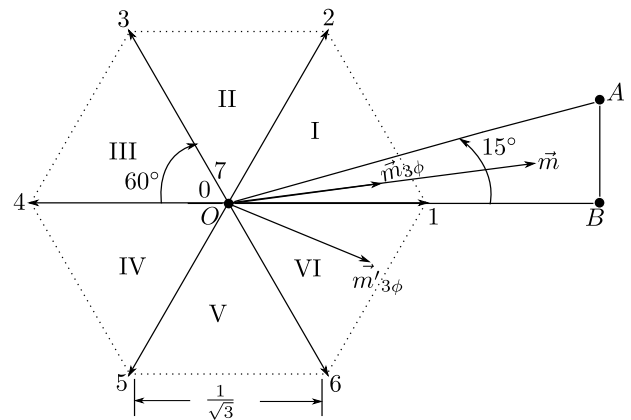

Fig. 3: $\vec{m}$ within $\triangle O A B$ and the corresponding $\vec{m}_{3 \phi}$ and $\vec{m}_{3 \phi}^{\prime}$

$z \in\{0,7\}$. Sector numbers in Fig. 2a are surrounded by ovalshaped curves. Modulation in the remaining sectors will be similar to sector-1.

$$
\begin{aligned}
& X_{i} \triangleq \frac{1}{\sqrt{3}}\left[\begin{array}{cccccc}
1 & -\frac{1}{2} & -\frac{1}{2} & \frac{\sqrt{3}}{2} & -\frac{\sqrt{3}}{2} & 0 \\
0 & \frac{\sqrt{3}}{2} & -\frac{\sqrt{3}}{2} & \frac{1}{2} & \frac{1}{2} & -1 \\
1 & -\frac{1}{2} & -\frac{1}{2} & -\frac{\sqrt{3}}{2} & \frac{\sqrt{3}}{2} & 0 \\
0 & -\frac{\sqrt{3}}{2} & \frac{\sqrt{3}}{2} & \frac{1}{2} & \frac{1}{2} & -1 \\
1 & 1 & 1 & 0 & 0 & 0 \\
0 & 0 & 0 & 1 & 1 & 1
\end{array}\right] X_{j} \\
& X_{i}=\underbrace{\left[\begin{array}{llllll}
x_{\alpha} & x_{\beta} & x_{z_{1}} & x_{z_{2}} & x_{o_{1}} & x_{o_{2}}
\end{array}\right]^{T}}_{T} \\
& X_{j}=\left[\begin{array}{llllll}
x_{a} & x_{b} & x_{c} & x_{a^{\prime}} & x_{b^{\prime}} & x_{c^{\prime}}
\end{array}\right]^{T}
\end{aligned}
$$

Linear modulation techniques (LMTs) of ASPM synthesizea) the desired reference voltage vector, $V_{\text {ref }} \triangleq \bar{v}_{\alpha}+j \bar{v}_{\beta}$, in $\alpha-\beta$ plane as it is solely responsible for electromagnetic energy transfer; b) zero average voltage vector in non-energy transferring $z_{1}-z_{2}$ plane in order to avoid unwanted average current and associated copper loss resulted due to the low impedance in this plane, i.e., $\bar{v}_{z_{1}}+j \bar{v}_{z_{2}}=0$. Here, bar represents average over a carrier cycle. To generalize the analysis, all lengths in subsequent figures are normalized with respect to DC-bus voltage $V_{D C}$, and the reference voltage vector is also normalized and denoted by $\vec{m}$. Here, $\vec{m} \triangleq m_{\alpha}+j m_{\beta}$ and $m_{\alpha, \beta}=\frac{\bar{v}_{\alpha, \beta}}{V_{D C}}$. In general, the synthesizable $\vec{m}$ can lie anywhere within a do-decagon structure obtained by joining the tips of the largest vectors in $\alpha-\beta$ plane and $D_{1}$ of Fig. 2a shows part of this do-decagon. But, [12] shows that synthesizable $\vec{m}$ of LMTs of ASPM can lie within another smaller do-decagon structure, $D_{2}$, due to the constraint of zero average vector synthesis in $z_{1}-z_{2}$. The linear modulation region within sector-1, $\triangle O A B$, is separately shown in Fig. 3, which is a right angle triangle with base length of unity and height of $\tan 15^{\circ}$.

$$
\begin{aligned}
& \quad \vec{m}_{3 \phi}=\frac{1}{2} \vec{m} ; \quad \vec{m}_{3 \phi}^{\prime}=\frac{1}{2} \vec{m} e^{-j 30^{\circ}} \\
& \vec{x}_{3 \phi}=\frac{1}{\sqrt{3}}\left[\left(x_{a}-\frac{x_{b}}{2}-\frac{x_{c}}{2}\right)+j \frac{\sqrt{3}}{2}\left(x_{b}-x_{c}\right)\right]
\end{aligned}
$$

[12] has shown that synthesis of $\vec{m}$ in $\alpha-\beta$ and zero in $z_{1}-z_{2}$ is mathematically equivalent to modulating the two $3 \phi$ inverters with reference voltage vectors as given in (2), where $\vec{m}_{3 \phi}$ and $\vec{m}_{3 \phi}^{\prime}$ are the reference voltage vectors of Inverter1 and Inverter-2, respectively. These $3 \phi$ voltage vectors are obtained by applying Clarke's $3 \phi-2 \phi$ transformation, as given in (3), on $3 \phi$ average line-neutral voltages. With respect to this transformation, the mapping of the line-neutral voltages generated by 8 switching states (given in Table I) of $3 \phi$ inverter is shown in Fig. 3.

Finding all possible linear modulation techniques when $\vec{m} \in$ $\triangle O A B$ is equivalent to finding all possible ways to synthesize $\vec{m}_{3 \phi}$ and $\vec{m}_{3 \phi}^{\prime}$ in per $3 \phi$ inverter basis. The intermediate steps, which are followed to derive these techniques from the given $\vec{m}$ in sector- 1 , are discussed below.

\section{A. Determine vector-sets per $3 \phi$ inverter and their corre- sponding duty-ratios}

When $\vec{m} \in \triangle O A B, 0^{\circ} \leq \angle \vec{m} \leq 15^{\circ}$. Therefore, according to (2), $0^{\circ} \leq \angle \vec{m}_{3 \phi} \leq 15^{\circ}$ and $-30^{\circ} \leq \angle \vec{m}_{3 \phi}^{\prime} \leq-15^{\circ}$. Hence, $\vec{m}_{3 \phi}$ and $\vec{m}_{3 \phi}^{\prime}$ lie within sector-I and sector-VI, respectively, in complex plane of $3 \phi$ inverter, as shown in Fig. 3. It is known from the modulation techniques of the conventional $3 \phi$ inverter that atleast three distinct vectors need to be applied per inverter basis in order to generate these reference voltage vectors. Two very popular choices are-i) nearest two active vectors and one zero vector, ii) nearest three active vectors. Therefore, Inverter-1 can use vector-set $\{z, 1,2\}$ or $\{6,1,2\}$ in order to synthesize $\vec{m}_{3 \phi}$, where $z \in\{0,7\}$. Similarly, Inverter2 can use vector-set $\left\{z^{\prime}, 1^{\prime}, 6^{\prime}\right\}$ or $\left\{6^{\prime}, 1^{\prime}, 2^{\prime}\right\}$ to synthesize $\vec{m}^{\prime}{ }_{3 \phi}$. The switching states of Inverter- 2 are labelled by primes.

$$
\begin{gathered}
d_{1} \frac{1}{\sqrt{3}}+d_{2} \frac{1}{\sqrt{3}} e^{j 60^{\circ}}=\vec{m}_{3 \phi} \triangleq \frac{1}{2}\left(m_{\alpha}+j m_{\beta}\right) ; \quad d_{1}+d_{2}+d_{z}=1 \\
m_{\alpha} \geq \frac{1}{\sqrt{3}} \quad \text { (5a) } \quad \sqrt{3} m_{\alpha}+m_{\beta} \geq \frac{2}{\sqrt{3}}
\end{gathered}
$$

The duty-ratios of each of the vectors of these vector-sets can be found by solving the average voltage equations. For example, $d_{z}, d_{1}$, and $d_{2}$, duty-ratios of the vectors of vectorset $\{z, 1,2\}$, can be found by solving (4). These are functions of $m_{\alpha}$ and $m_{\beta}$. Similarly, duty-ratios of the vectors of other vector-sets can be found. The expressions of these duty-ratios of the above four vector-sets are given in Table II. Note, $\{6,1,2\}$ and $\left\{6^{\prime}, 1^{\prime}, 2^{\prime}\right\}$ can't be used for the entire $\triangle O A B$ as $d 1$ of $\{6,1,2\}$ and $d 1^{\prime}$ of $\left\{6^{\prime}, 1^{\prime}, 2^{\prime}\right\}$ become negative for some part of $\triangle O A B$. The regions, where $\{6,1,2\}$ and $\left\{6^{\prime}, 1^{\prime}, 2^{\prime}\right\}$ can be used, are identified by solving the inequalities that all the duty-ratios are greater than equal to zero and they are given in (5a) and (5b), respectively.

B. Determine the sequences of $3 \phi$ inverters corresponding to each vector-set

The sequence design will be guided by rules $\boldsymbol{R} \mathbf{1}$ and $\boldsymbol{R} 2$, as given in the Introduction. $\mathbf{R} 2$ ensures that all the vectors within the given vector-set need to be applied atleast once within carrier half-period, $\frac{T_{s}}{2}$. It can be shown that all possible switching sequences of vector-set $\{z, 1,2\}$, which obey the above two rules, can be generalized as $0-1-2-7-2-1-0$ within $T_{s}$ or $0-1-2-7$ within $\frac{T_{s}}{2}$. From now onwards, we will only consider the vector-sequence within $\frac{T_{s}}{2}$ as the other half is mirror symmetric. Similarly, vector-set $\left\{z^{\prime}, 1^{\prime}, 6^{\prime}\right\},\{6,1,2\}$, and $\left\{6^{\prime}, 1^{\prime}, 2^{\prime}\right\}$ can be generalized as $0^{\prime}-1^{\prime}-6^{\prime}-7^{\prime}, 6-1-2$, and $6^{\prime}-1^{\prime}-2^{\prime}$, respectively. 
TABLE II: Duty ratios of the switching states of four vector-sets

\begin{tabular}{c|c}
\multicolumn{2}{c}{ Vector-set $\{z, 1,2\}$} \\
$d 1$ & $\frac{\sqrt{3}}{2} m_{\alpha}-\frac{1}{2} m_{\beta}$ \\
\hline$d 2$ & $m_{\beta}$ \\
\hline$d z$ & $1-\frac{\sqrt{3}}{2} m_{\alpha}-\frac{1}{2} m_{\beta}$
\end{tabular}

\begin{tabular}{c|c}
\multicolumn{2}{c}{ Vector-set $\{6,1,2\}$} \\
$d 1$ & $\sqrt{3} m_{\alpha}-1$ \\
\hline$d 2$ & $1-\frac{\sqrt{3}}{2} m_{\alpha}+\frac{1}{2} m_{\beta}$ \\
\hline$d 6$ & $1-\frac{\sqrt{3}}{2} m_{\alpha}-\frac{1}{2} m_{\beta}$
\end{tabular}

\begin{tabular}{c|c}
\multicolumn{2}{c}{ Vector-set $\left\{z^{\prime}, 1^{\prime}, 6^{\prime}\right\}$} \\
$d 1^{\prime}$ & $\frac{1}{2} m_{\alpha}+\frac{\sqrt{3}}{2} m_{\beta}$ \\
\hline$d 6^{\prime}$ & $\frac{1}{2} m_{\alpha}-\frac{\sqrt{3}}{2} m_{\beta}$ \\
\hline$d z^{\prime}$ & $1-m_{\alpha}$
\end{tabular}

Vector-set $\left\{6^{\prime}, 1^{\prime}, 2^{\prime}\right\}$
\begin{tabular}{c|c}
$d 1^{\prime}$ & $\frac{3}{2} m_{\alpha}+\frac{\sqrt{3}}{2} m_{\beta}-1$ \\
\hline$d 2^{\prime}$ & $1-m_{\alpha}$ \\
\hline$d 6^{\prime}$ & $1-\frac{1}{2} m_{\alpha}-\frac{\sqrt{3}}{2} m_{\beta}$
\end{tabular}

TABLE III: 8 possible sequence-groups when $\vec{m} \in \triangle O A B$

\begin{tabular}{|c|c|c|c|c|c|}
\hline Case & Sequence over $\frac{T_{s}}{2}$ & $k_{f}$ & Case & Sequence over $\frac{T_{s}}{2}$ & $k_{f}$ \\
\hline S1 & $\begin{array}{l}\overbrace{0}^{x}-1-2-\overbrace{7}^{1-x} \\
\underbrace{0^{\prime}}_{y}-1^{\prime}-6^{\prime}-\underbrace{7^{\prime}}_{1-y}\end{array}$ & \multirow{2}{*}{$\begin{array}{l}1 ; x, y \in(0,1) \\
\frac{2}{3} ; x, y \in\{0,1\} \\
\frac{5}{6} ; \text { o.W. }\end{array}$} & S5 & $\begin{array}{r}6-1-2 \\
\underbrace{0^{\prime}}_{y}-1^{\prime}-6^{\prime}-\underbrace{7^{\prime}}_{1-y}\end{array}$ & \multirow{2}{*}{$k_{f}=\left\{\begin{array}{l}\frac{5}{6} ; 0<y<1 \\
\frac{2}{3} ; y \in\{0,1\}\end{array}\right.$} \\
\hline S2 & $\begin{array}{l}\overbrace{0}^{x}-1-2-\overbrace{7}^{1-x} \\
\underbrace{7^{\prime}}_{y}-6^{\prime}-1^{\prime}-\underbrace{0^{\prime}}_{1-y}\end{array}$ & & S6 & $\begin{array}{r}6-1-2 \\
\underbrace{7^{\prime}}_{y}-6^{\prime}-1^{\prime}-\underbrace{0^{\prime}}_{1-y}\end{array}$ & \\
\hline S3 & $\begin{array}{r}\overbrace{0}^{x}-1-2-\overbrace{7}^{1-x} \\
6^{\prime}-1^{\prime}-2^{\prime} \\
\end{array}$ & \multirow{2}{*}{$k_{f}=\left\{\begin{array}{l}\frac{5}{6} ; 0<x<1 \\
\frac{2}{3} ; x \in\{0,1\}\end{array}\right.$} & S7 & $\begin{array}{r}6-1-2 \\
6^{\prime}-1^{\prime}-2^{\prime} \\
\end{array}$ & \multirow[t]{2}{*}{$k_{f}=\frac{2}{3}$} \\
\hline S4 & $\begin{array}{r}\overbrace{0}^{x}-1-2-\overbrace{7}^{1-x} \\
2^{\prime}-1^{\prime}-6^{\prime}\end{array}$ & & S8 & $\begin{array}{r}6-1-2 \\
2^{\prime}-1^{\prime}-6^{\prime}\end{array}$ & \\
\hline
\end{tabular}

C. Sequence grouping of $6 \phi$ inverter

As each of Inverter- 1 and Inverter- 2 has two sequences to synthesize $\vec{m}_{3 \phi}$ and $\vec{m}_{3 \phi}^{\prime}$, respectively, $\vec{m}$ in $\alpha-\beta$ can be synthesized by using $2 \times 2=4$ different combinations of $3 \phi$ sequences. For each of these four combinations, reversal of one sequence with respect to the other gives 2 different arrangements. Therefore, total 8 sequence-groups can occur and Table III shows these 8 groups. These are labelled as S1 to S8. As sequences $0-1-2-7$ and $0^{\prime}-1^{\prime}-6^{\prime}-7^{\prime}$ can be used to synthesize $\vec{m}$ within the entire $\triangle O A B$, S1 and $\mathrm{S} 2$ can be applied for the entire $\triangle O A B$. Similarly, from our previous discussion it is obvious that $\mathrm{S} 3$ and $\mathrm{S} 4$ are applicable in the region within $\triangle O A B$ where condition (5b) is satisfied; $\mathrm{S} 5$ and $\mathrm{S} 6$ are applicable where (5a) is satisfied and S7 and S8 are applicable where both (5a) and (5b) are satisfied. From $3 \phi$ inverter literature, it is known that the current-ripple is function of zero-vector splitting, [9], [15], [16]. Therefore, the zero-vector dwell-times are split as $x,(1-x)$ (for Inverter-1) and $y,(1-y)$ (for Inverter-2) between redundant zero states 0 and 7 to study the ripple performances in next section. Here, $x$ and $y$ are non-negative fractions. Note, $x, y \in\{0,1\}$ results into conventional bus-clamping PWM of $3 \phi$ inverter.

Let's define $k_{f}$ as the ratio of total number of switching (combining turn-on and turn-off) over $T_{s}$ and total number of legs (6 in this case). As each change of state is accompanied by one transition, $k_{f}=\frac{m+n-2}{6}$ when the two $3 \phi$ inverters apply $m$ and $n$ number of states, respectively, within $\frac{T_{s}}{2}$. From this discussion, one can see that $k_{f}$ is function of $x$ and $y$ and this function is shown in Table III. As we will see later that $k_{f}$ plays an important role in current-ripple comparison of different techniques with same average switching frequency.

\section{Determine the vector sequence and corresponding duty} ratios of $6 \phi$ inverter

The objective of this paper is to find the best technique in terms of current-ripple out of infinite number of possible

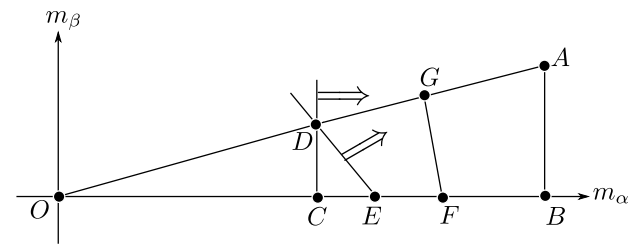

Fig. 4: S7 applied within $\triangle O A B$

techniques, grouped as $\mathrm{S} 1$ to $\mathrm{S} 8$, for $\vec{m} \in \triangle O A B$. This infinite possibility arises due to the variability exists in the choice of $x$ and $y$ (S1-S6). [6] shows that the estimation of current-ripple requires the sequence of application of $6 \phi$ switching states (to know the corresponding voltage vectors in both $\alpha-\beta$ and $z_{1}-z_{2}$ planes) and their corresponding dwell times. Given any selection, $S 1$ to $S 8$, this sequence and the dwell times of the states depend upon position of $\vec{m}$, choices of $x$ and $y$ (in case of S1 to S6).

To explain the above, let's consider case S7 over $\frac{T_{s}}{2}$. As S7 involves vector-sets $\{6,1,2\}$ and $\left\{6^{\prime}, 1^{\prime}, 2^{\prime}\right\}$, it can be applied in part of $\triangle O A B$ where both (5a) and (5b) are satisfied. The straight lines with equality in (5a) and (5b) are plotted as $C D$ and $E D$ in Fig. 4. Double-arrow marks on these lines show the part of $\triangle O A B$ where conditions (5a) and (5b) are satisfied and therefore, $\square E D A B$ is the region where $\mathrm{S} 7$ can be applied. Fig. 5 shows all six probable cases of S7, where states of Inverter- 1 and Inverter- 2 are placed in top and bottom, respectively. From Fig. 5, 6 $\phi$ sequences and the dwell time of each switching state corresponding to a sequence can be determined. For example, sequence of case $(f)$ of Fig. 5 is $\left(6,6^{\prime}\right)-\left(1,6^{\prime}\right)-\left(1,1^{\prime}\right)-\left(2,1^{\prime}\right)-\left(2,2^{\prime}\right)$, as shown in Fig. 6. The corresponding duty-ratios of these states with respect to $\frac{T_{s}}{2}$ are $D 1=d 6, D 2=d 6^{\prime}-d 6, D 3=d 1-\left(d 6^{\prime}-d 6\right), D 4=$ $d 2-d 2^{\prime}$, and, $D 5=d 2^{\prime}$, respectively, where the duty values, $d k$ and $d k^{\prime}, k=1,2,6$, correspond to vector-sets $\{6,1,2\}$ and $\left\{6^{\prime}, 1^{\prime}, 2^{\prime}\right\}$, respectively, of Table II. Similarly, one can find the sequences for other cases of S7, which are tabulated in Table IV. For a given $\vec{m} \triangleq m_{\alpha}+j m_{\beta}$, one of these six 

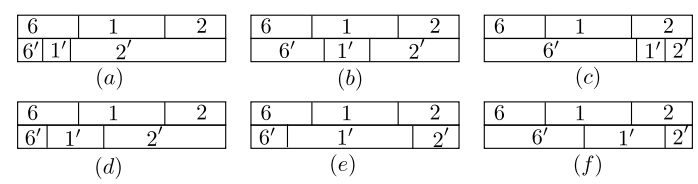

Fig. 5: Six possible arrangements of sequencegroup $\mathrm{S} 7$

\begin{tabular}{|c|c|c|c|c|}
\hline$D 1$ & $D 2$ & D3 & $D 4$ & $D 5$ \\
\hline 6 & 1 & & 2 & \\
\hline $6^{\prime}$ & & 1 & & $2^{\prime}$ \\
\hline$\left(6,6^{\prime}\right)$ & $\left(1,6^{\prime}\right)$ & & $(2,1$ & $\left(2,2^{\prime}\right)$ \\
\hline
\end{tabular}

Fig. 6: Sequence of Fig. $5 \mathrm{f}$

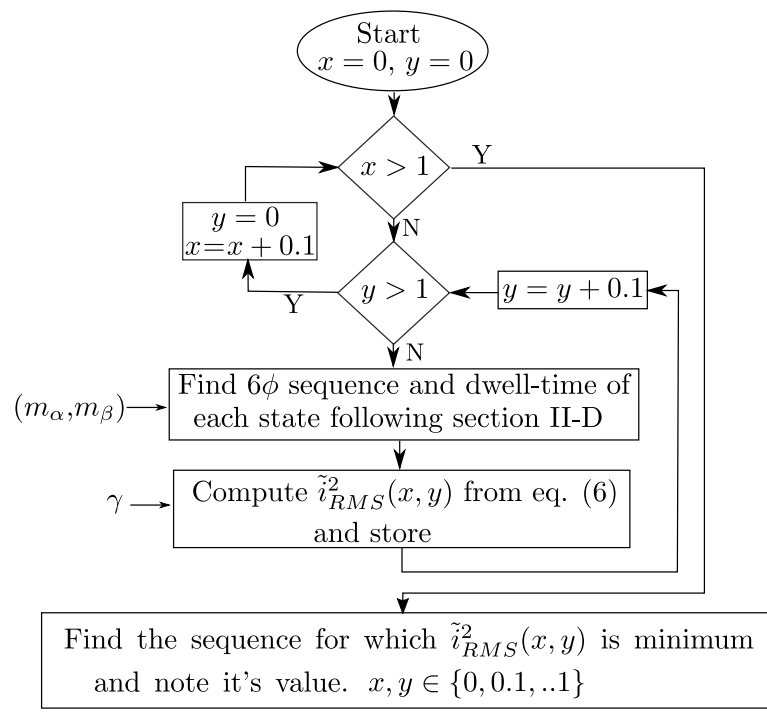

Fig. 7: Algorithm to find the optimal technique of sequencegroups S1 and S2

cases can occur as the duty ratios are defined by $m_{\alpha}$ and $m_{\beta}$, as given in Table II.

Number of cases for other sequence-groups can also be found in the similar manner and are given in Table V. It is important to mention here that all these possibilities may not arise in case of $\vec{m} \in \Delta O A B$. For example, it is possible to show that only two sequences, cases $(c)$ and $(f)$ of Table IV, can occur within $\square E D A B$ from the six possible sequences of S7. These two sequences are applied in $\square E D G F$ and $\square F G A B$, respectively, where the equation of $F G$ in Fig. 4 is $m_{\alpha}+\frac{1}{2+\sqrt{3}} m_{\beta}=\frac{2}{1+\sqrt{3}}$.

\section{Determination OF TEChNiQue(s) With OPTIMAL} CURRENT-RIPPLE PERFORMANCE

To minimize RMS ripple current over a line-cycle, this paper proposes to minimize the ripple RMS current at every carrier cycle. The total RMS ripple current of all six phases over a carrier cycle can be derived after following the steps given in [6]. The expression of this RMS current, $\tilde{i}_{R M S}$, is given in (6). Here, RMS ripple current of $j^{t h}$ phase, $\tilde{i}_{j, R M S}$, and $\tilde{i}_{R M S}$ are per-unitized with respect to base current $\frac{V_{D C} T_{s w}}{L_{\alpha \beta}} \cdot \gamma$ is a machine parameter which is defined as $\gamma \triangleq \frac{L_{\alpha \beta}}{L_{z_{1} z_{2}}} ; L_{\alpha \beta}$ and $L_{z_{1} z_{2}}$ are the high-frequency equivalent circuit inductances in $\alpha-\beta$ and $z_{1}-z_{2}$ planes, respectively. $\tilde{\lambda}_{\alpha \beta}$ and $\tilde{\lambda}_{z_{1} z_{2}}$ are the ripple RMS fluxes in these two planes. In (6), the factor $k_{f} \triangleq \frac{T_{s}}{T_{s w}}$ is introduced to keep average switching frequency,
TABLE V: Number of probable sequences of S1-S8

$\left(6,6^{\prime}\right)-\left(6,1^{\prime}\right)-\left(6,2^{\prime}\right)-\left(1,2^{\prime}\right)-\left(2,2^{\prime}\right)$

$\left(6,6^{\prime}\right)-\left(1,6^{\prime}\right)-\left(1,1^{\prime}\right)-\left(1,2^{\prime}\right)-\left(2,2^{\prime}\right)$

$\left(6,6^{\prime}\right)-\left(1,6^{\prime}\right)-\left(2,6^{\prime}\right)-\left(2,1^{\prime}\right)-\left(2,2^{\prime}\right)$

$\left(6,6^{\prime}\right)-\left(6,1^{\prime}\right)-\left(1,1^{\prime}\right)-\left(1,2^{\prime}\right)-\left(2,2^{\prime}\right)$

$\left(6,6^{\prime}\right)-\left(6,1^{\prime}\right)-\left(1,1^{\prime}\right)-\left(2,1^{\prime}\right)-\left(2,2^{\prime}\right)$

$\left(6,6^{\prime}\right)-\left(1,6^{\prime}\right)-\left(1,1^{\prime}\right)-\left(2,1^{\prime}\right)-\left(2,2^{\prime}\right)$

\begin{tabular}{c|c} 
Case & No. of Sequences \\
\hline S1, S2 & 20 \\
S3-S6 & 10 \\
S7, S8 & 6
\end{tabular}

$F_{s w} \triangleq \frac{1}{T_{s w}}$, same for all techniques during comparison. It can be seen that $\tilde{i}_{R M S}$ depends only on modulation technique and $\gamma$. The parameter $\gamma$ is a strong function of winding pitch-factor and geometry of the slot of the machine, [17]. In this work, the feasible range of $\gamma$ is considered to be 1.5 to 10 .

$\tilde{i}_{R M S}^{2} \triangleq \sum_{j=a}^{c^{\prime}} \tilde{i}_{j, R M S}^{2}=k_{f}^{2}\left(\tilde{\lambda}_{\alpha \beta}^{2}\left(m_{\alpha}, m_{\beta}\right)+\gamma^{2} \tilde{\lambda}_{z_{1} z_{2}}^{2}\left(m_{\alpha}, m_{\beta}\right)\right)$

With the above formulation, the optimal technique is found numerically, rather than analytically, due to the complex nature of the problem. The algorithm to find the optimal technique, for a given $\vec{m} \triangleq m_{\alpha}+j m_{\beta}$ and machine parameter $\gamma$, is explained as follows.

1) Step-1: The optimal sequence of a particular sequencegroup is identified and the corresponding $\tilde{i}_{R M S}^{2}$ is recorded. A flowchart of this step is given in Fig. 7 for sequence-groups $\mathrm{S} 1$ and $\mathrm{S} 2$. Here, values of $x$ and $y$ are varied between 0 and 1 with spacing of 0.1 between two consecutive values. Similar procedure can be followed to find the optimal sequence and corresponding optimal ripple RMS currents of sequence groups S3 to S6 (either $x$ or $y$ is varying), $\mathrm{S} 7$ and $\mathrm{S} 8$ (only one sequence).

2) Step-2: Based on the conditions given in (5), there could be maximum of 8 optimal sequences, one coming form each sequence groups, S1 to S8. From these 8 (or less than 8) sequences, find the sequence for which $\tilde{i}_{R M S}^{2}$ is minimum. For the given $\vec{m}$ and $\gamma$, this sequence is the optimal sequence out of all possible sequences which follow rules $\boldsymbol{R} \mathbf{1}$ and $\boldsymbol{R} \mathbf{2}$.

The above algorithm is run for a range of $\vec{m} \in \triangle O A B$ and for feasible range of $\gamma$. Fig. 8 shows region-wise optimal techniques for three values of $\gamma$ within $\triangle O A B$. It is interesting to notice that only five techniques, out of so many possibilities, are optimal for most of the region of $\triangle O A B$ for $1.5 \leq \gamma \leq 10$. These are labelled as Optimal Linear Technique-1/OLT-1 to OLT-5. Table VI tabulates $6 \phi$ sequences, type of $\alpha-\beta$ plane vectors used, and the sequence-group and corresponding $x, y$ values of these techniques. Please refer these states from Fig. $2 \mathrm{a}$, where the non-zero voltage vectors are of three different lengths- large (L), medium (M) and small (S). The zero vector, $\left(z, z^{\prime}\right)$ where $z \in\{0,7\}$, is denoted by Z. Other optimal techniques, like, S2, $x=0.6, y=0.5$ and S2, $x=0.5$, $y=0.4$, show optimality for very small region at $1.5 \leq \gamma \leq 2$ and are neglected for practical implementation. One should note that OLT-1 is same as existing C6 $\phi S V P W M 24-C$, [8]. This is the only optimal technique which exists in the literature.

Let's define $\psi_{\alpha \beta}^{2} \triangleq k_{f}^{2} \tilde{\lambda}_{\alpha \beta}^{2}$ and $\psi_{z_{1} z_{2}}^{2} \triangleq k_{f}^{2} \tilde{\lambda}_{z_{1} z_{2}}^{2}$. The analytical closed-from expressions of $\psi_{\alpha \beta}^{2}$ and $\psi_{z_{1} z_{2}}^{2}$ of all of the above optimal vector-sequences are derived and these 


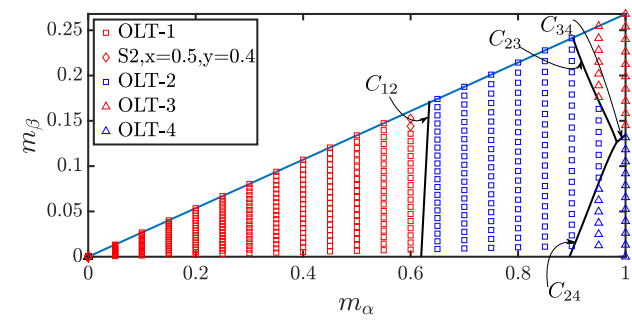

(a) $\gamma=2$

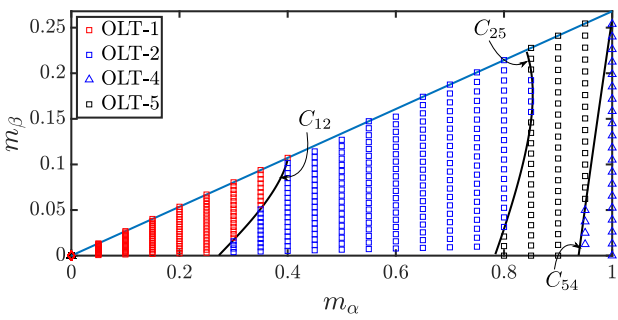

(b) $\gamma=6$

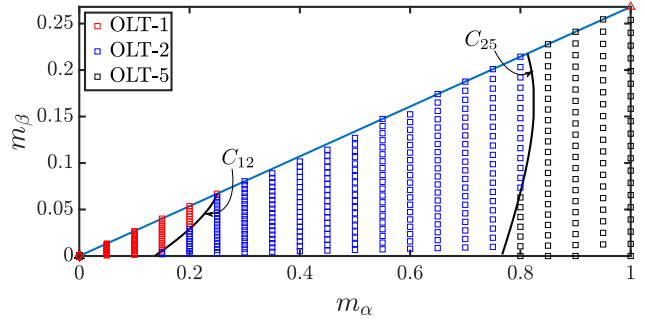

(c) $\gamma=10$

Fig. 8: Region-wise optimal techniques for different values of $\gamma$

TABLE VI: Optimal five Linear PWM techniques of ASPM

\begin{tabular}{cccc}
\hline \hline OLTs & Switching sequence over $\frac{T_{s}}{2}$ & Vector type & Sequence-group, $x, y$ \\
\hline OLT-1 & $\left(0,7^{\prime}\right)-\left(0,6^{\prime}\right)-\left(1,6^{\prime}\right)-\left(1,1^{\prime}\right)-\left(2,1^{\prime}\right)-\left(7,1^{\prime}\right)-\left(7,0^{\prime}\right)$ & $3 \mathrm{~L}+2 \mathrm{~S}+2 \mathrm{Z}$ & $\mathrm{S} 2, x=0.5, y=0.5$ \\
\hline OLT-2 & $\left(0,7^{\prime}\right)-\left(0,6^{\prime}\right)-\left(1,6^{\prime}\right)-\left(1,1^{\prime}\right)-\left(2,1^{\prime}\right)$ & $3 \mathrm{~L}+1 \mathrm{~S}+1 \mathrm{Z}$ & $\mathrm{S} 2, x=1, y=1$ \\
\hline OLT-3 & $\left(0,6^{\prime}\right)-\left(1,6^{\prime}\right)-\left(1,1^{\prime}\right)-\left(2,1^{\prime}\right)-\left(2,0^{\prime}\right)$ & $3 \mathrm{~L}+2 \mathrm{~S}$ & $\mathrm{~S} 2, x=1, y=0$ \\
\hline OLT-4 & $\left(6,6^{\prime}\right)-\left(1,6^{\prime}\right)-\left(1,1^{\prime}\right)-\left(2,1^{\prime}\right)-\left(2,0^{\prime}\right)$ & 4L+1S & $\mathrm{S} 6, y=0$ \\
\hline OLT-5 & $\left(6,6^{\prime}\right)-\left(1,6^{\prime}\right)-\left(1,1^{\prime}\right)-\left(2,1^{\prime}\right)-\left(2,2^{\prime}\right)$ & $5 \mathrm{~L}$ & $\mathrm{~S} 7$ \\
\hline \hline
\end{tabular}

are fourth and third order polynomials, respectively, of two variables, $m_{\alpha}$ and $m_{\beta}$. The generalized analytical expressions of $\psi_{\alpha \beta}^{2}$ and $\psi_{z_{1} z_{2}}^{2}$ is given in (7) and Table VII lists the coefficients of these polynomial expressions of five optimal techniques. Few important observations of this study are as follows.

- Based on the set of optimal techniques, that one needs to consider for the entire $\triangle O A B$, the feasible value of $\gamma$ can fall in three ranges-

i) low $(1.5 \leq \gamma \leq 3.5)$, where OLT- 1 to OLT-4 are used.

ii) medium (3.5 $\leq \gamma \leq 8)$, where OLT-1, OLT-2, OLT-4, and OLT-5 are used.

iii) high $(8 \leq \gamma \leq 10)$, where OLT-1, OLT-2, and OLT-5 are used.

- OLT-1 shows optimality at low voltage gain; OLT-2 for medium voltage gain; and OLT-3, OLT-4 and OLT-5 at higher voltage gain.

\section{Proposed Hybrid Optimal Technique}

Based on the machine parameter, $\gamma$, falling in one of the three ranges, one can easily identify the optimal techniques in the entire region of a sector and thereafter, implement a optimal technique at every carrier-cycle for the given $\vec{m}$. To know the boundary between the optimal regions of $i^{\text {th }}$ and $j^{\text {th }}$ optimal techniques, one needs to equate their carrier-cycle
TABLE VIII: $k_{f}$ of different techniques

\begin{tabular}{cc}
\hline Technique & $k_{f}$ \\
\hline D6 $\phi$ SVPWM24-B2 & $\frac{2}{3}$ \\
STPWM, ZS6PWM, DZIPWM & 1 \\
C6 $\phi$ SVPWM24-C / OLT-1 & 1 \\
OLT-2, OLT-3, OLT-4, OLT-5 & $\frac{2}{3}$ \\
\hline
\end{tabular}

RMS ripple current as given in (6), i.e., $\left(\psi_{\alpha \beta}^{2}+\gamma^{2} \psi_{z_{1} z_{2}}^{2}\right)_{i}=$ $\left(\psi_{\alpha \beta}^{2}+\gamma^{2} \psi_{z_{1} z_{2}}^{2}\right)_{j}$ and these expressions are given in (7) and Table VII as the function of $m_{\alpha}$ and $m_{\beta}$. The boundary between $i^{\text {th }}$ and $j^{\text {th }}$ optimal techniques, denoted by $C_{i j}$, $i, j \in\{1,2, . .5\}$, is shown in Fig. 8 .

This proposed hybrid optimal technique is compared in Fig. 9 with the existing LMTs of ASPM, which are discussed in Introduction. For this comparison, the carrier-cycle ripple current are integrated over a line-cycle for sinusoidal excitation in $\alpha-\beta$ with $\vec{m}=\sqrt{3} M_{I} e^{j \omega_{o} t}$ and zero average voltage excitation in $z_{1}-z_{2}$ to get the line-cycle total ripple current RMS as function of $M_{I}$. Applying inverse transformation of $T$ on these transformed domain voltages, one can obtain the phase voltages as $\bar{v}_{a o}=M_{I} V_{D C} \cos \omega_{o} t$, $\bar{v}_{a^{\prime} o^{\prime}}=M_{I} V_{D C} \cos \left(\omega_{o} t-\frac{\pi}{6}\right) ; \bar{v}_{b o / b^{\prime} o^{\prime}}$ and $\bar{v}_{c o / c^{\prime} o^{\prime}}$ are $120^{\circ}$ and $240^{\circ}$ phase-shifted with respect to $\bar{v}_{a o / a^{\prime} o^{\prime}}$. Here, $M_{I}$ is modulation index which is defined as the ratio of peak line-neutral voltage and DC-bus voltage. The line-cycle RMS of the ripple current of each phase, $\tilde{I}_{R M S}$, can be obtained as (8) from (6), which is also per-unitized with respect to

$$
\begin{aligned}
\psi_{\alpha \beta / z_{1} z_{2}}^{2}\left(m_{\alpha}, m_{\beta}\right)= & \left(C_{1} m_{\alpha}^{4}+C_{2} m_{\alpha}^{3} m_{\beta}+C_{3} m_{\alpha}^{2} m_{\beta}^{2}+C_{4} m_{\alpha} m_{\beta}^{3}+C_{5} m_{\beta}^{4}\right)+\left(C_{6} m_{\alpha}^{3}+C_{7} m_{\alpha}^{2} m_{\beta}+C_{8} m_{\alpha} m_{\beta}^{2}+C_{9} m_{\beta}^{3}\right) \\
& +\left(C_{10} m_{\alpha}^{2}+C_{11} m_{\alpha} m_{\beta}+C_{12} m_{\beta}^{2}\right)+\left(C_{13} m_{\alpha}+C_{14} m_{\beta}\right)+C_{15}
\end{aligned}
$$

\begin{tabular}{|c|c|c|c|c|c|c|c|c|c|c|c|c|c|c|c|c|}
\hline Techniques & & $C_{1}$ & $C_{2}$ & $C_{3}$ & $C_{4}$ & $C_{5}$ & $C_{6}$ & $C_{7}$ & $C_{8}$ & $C_{9}$ & $C_{10}$ & $C_{11}$ & $C_{12}$ & $C_{13}$ & $C_{14}$ & $C_{15}$ \\
\hline \multirow{2}{*}{ OLT-1 } & $\psi_{\alpha \beta}^{2}$ & 18.2 & 0 & 46.9 & -36.1 & 49.5 & $\begin{array}{l}-37.2 \\
\end{array}$ & 8.9 & -64.6 & -19.1 & 20.8 & 0 & 20.8 & 0 & 0 & 0 \\
\hline & $\psi_{z_{1} z_{2}}^{2}$ & 0 & 0 & 0 & 0 & 0 & 1.8 & -8.9 & 15.3 & 5.2 & 0 & 0 & 0 & 0 & 0 & 0 \\
\hline \multirow{2}{*}{ OLT-2 } & $\psi_{\alpha \beta}^{2}$ & 32.4 & 16 & 27.8 & 16 & 32.4 & -68.5 & -13.9 & -55.6 & -54.4 & 37 & 0 & 37 & 0 & 0 & 0 \\
\hline & $\psi_{z_{1} z_{2}}^{2}$ & 0 & 0 & 0 & 0 & 0 & 0.9 & -5.9 & 13.9 & -3.6 & 0 & 0 & 0 & 0 & 0 & 0 \\
\hline \multirow{2}{*}{ OLT-3 } & $\psi_{\alpha \beta}^{2}$ & 32.4 & 32.1 & 27.8 & -32.1 & 32.4 & -103.2 & -73.7 & -35.8 & -6.3 & 120.9 & 59.8 & 17.3 & -59.8 & -16 & -10.7 \\
\hline & $\psi_{z_{1} z_{2}}^{2}$ & 0 & 0 & 0 & 0 & 0 & 35.7 & 37.9 & -5.9 & -3.6 & -83.9 & -59.9 & 19.8 & 59.9 & 16 & -10.7 \\
\hline \multirow{2}{*}{ OLT-4 } & $\psi_{\alpha \beta}^{2}$ & 32.4 & 8 & 69.4 & -24.1 & 18.5 & -73.3 & -40.1 & -102.1 & 24.1 & 33.3 & 59.9 & 22.2 & 16 & -27.8 & -6.2 \\
\hline & $\psi_{z_{1}}^{2} z_{2}$ & 0 & 0 & 0 & 0 & 0 & $\begin{array}{ll}37.8 \\
\end{array}$ & $\begin{array}{l}32.1 \\
\end{array}$ & $\begin{array}{l}-3.7 \\
\end{array}$ & 0 & -79.6 & -59.9 & 5.5 & 48.1 & 27.8 & -6.2 \\
\hline \multirow{2}{*}{ OLT-5 } & $\psi_{\alpha \beta}^{2}$ & 32.4 & -8 & 69.4 & 24.1 & 18.5 & -27.8 & 28.4 & -80.2 & -24.1 & -83 & -48.1 & 0.4 & 103.7 & 27.8 & -23 \\
\hline & $\psi_{z_{1} z_{2}}^{2}$ & 0 & 0 & 0 & 0 & 0 & 13.9 & 11.7 & 2.1 & 0 & -28.1 & -16 & -0.3 & 16 & 4.3 & -1.7 \\
\hline
\end{tabular}

TABLE VII: Coefficients of $\psi_{\alpha \beta}^{2}\left(m_{\alpha}, m_{\beta}\right)$ and $\psi_{z_{1} z_{2}}^{2}\left(m_{\alpha}, m_{\beta}\right)$ of different techniques (multiplied by 1000) 


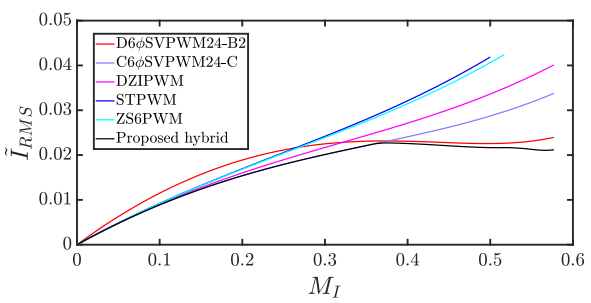

(a) $\gamma=2$

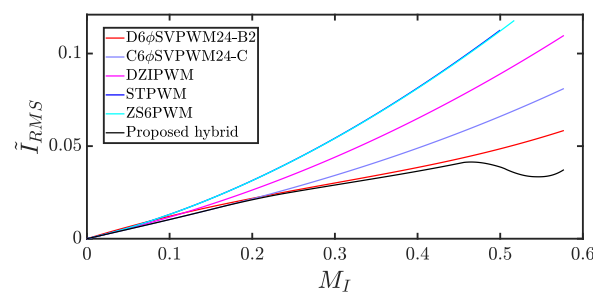

(b) $\gamma=6$

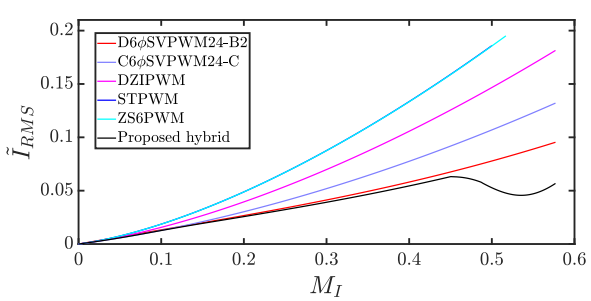

(c) $\gamma=10$

Fig. 9: Comparison of ripple current performances of five existing techniques and proposed hybrid technique

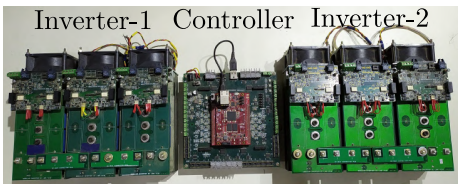

(a)

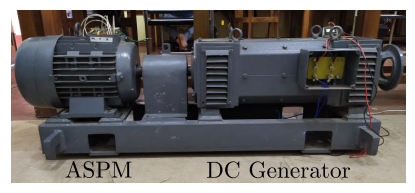

(b)
Fig. 10: Experimental set-up. (a) Six-phase inverter and controller. (b) ASPM coupled with DC generator.

base current $\frac{V_{D C} T_{s w}}{L_{\alpha \beta}}$. As $\tilde{i}_{R M S}$ denotes switching cycle RMS current combining all six phases, and total ripple RMS current seen by all six phases over a line-cycle is same, factor $\frac{1}{6}$ is introduced in (8) to obtain $\tilde{I}_{R M S}^{2}$ of each phase. For fair comparison, we have chosen five existing techniques which follow rules R1 and R2; these are- 1) D6 $\phi$ SVPWM24-B2, the best technique of [7], 2) C6 $\phi$ SVPWM24-C, 3) Three TINV based techniques (STPWM, DZIPWM, ZS6PWM). As all of the 12-sector based techniques of [6] disobey rule R1, they are not included in this comparison. In this comparison, $k_{f}$ values of different techniques are considered as given in Table VIII to keep the average switching frequency same. $\tilde{I}_{R M S}$ vs $M_{I}$ of five existing techniques and the proposed hybrid technique are plotted in Fig. 9 up to the maximum attainable $M_{I} \mathrm{~s}$, which are 0.5 and 0.517 for STPWM and ZS6PWM, respectively, and 0.577 for other cases. The plots are given for three values of $\gamma(2,6$, and 10), which represent the three ranges as discussed before.

$$
\tilde{I}_{R M S}^{2}\left(M_{I}\right)=\frac{1}{6}\left(\frac{1}{2 \pi} \int_{0}^{2 \pi} \tilde{i}_{R M S}^{2}\left(M_{I}, \omega_{o} t\right) d\left(\omega_{o} t\right)\right)
$$

Fig. 9 shows that $\tilde{I}_{R M S}$ of the proposed technique is the lowest among all of the techniques for the entire range of $M_{I}$ and for all values of $\gamma$. This proves the superiority of the proposed hybrid optimal technique over other existing techniques. As OLT-1 is same as C6 $\phi$ SVPWM24-C, at low $M_{I}$ the proposed hybrid technique follows the curve of C6 $\phi$ SVPWM24-C. At higher values of $\gamma$ and $M_{I}$, the improvement of the proposed hybrid technique compared to the existing techniques becomes more evident.

\section{Simulation AND EXPERIMENTAL VALIDATION}

Fig. 10b shows a 2-pole $5 \mathrm{~kW}$ asymmetrical $6 \phi$ induction machine, coupled with DC generator for loading purpose, which is used for experimental validation. The equivalent circuit parameters of this machine are given in Table IX. $\gamma$ of this full-pitched winding machine is 1.69. SKM75GB123D IGBT based $6 \phi$ inverter along with Zynq-7010 based controller card are shown in Fig. 10a. MATLAB-Simulink is used for simulation and the dynamic model of $6 \phi$ machine is implemented in Simulink.
TABLE IX: Equivalent Circuit Parameters of $6 \phi$ IM

\begin{tabular}{cc}
\hline Per phase stator and rotor resistances & $0.675 \Omega$ \\
Per phase magnetizing inductance & $0.186 \mathrm{H}$ \\
High-frequency $\alpha-\beta$ plane inductance, $L_{\alpha \beta}$ & $2.97 \mathrm{mH}$ \\
High-frequency $z_{1}-z_{2}$ plane inductance, $L_{z_{1}} z_{2}$ & $1.76 \mathrm{mH}$ \\
Ratio of inductances in $\alpha-\beta$ and $z_{1}-z_{2}, \gamma$ & 1.69 \\
\hline
\end{tabular}

Experiments and simulations are performed at nine values of $M_{I}$ between 0.2 and 0.577 , .viz $M_{I} \in$ $\{0.2,0.25,0.3,0.35,0.4,0.45,0.5,0.55,0.577\}$, with following operating conditions: $V_{D C}=300 \mathrm{~V} ; F_{s w}=8.33 \mathrm{kHz}$. For constant V/f operation, $M_{I}$ is changed along with output frequency, $f_{o}$, which results into $50 \mathrm{~Hz}$ operation at maximum $M_{I}$ (0.577). As the machine used in the experiment has $\gamma=1.69$ (belongs to low range), the hybrid optimal technique involves OLT-1 to OLT-4, as discussed in sectionIII. $k_{f}$ of OLT- 1 is 1 and $k_{f}$ of other OLTs is $\frac{2}{3}$. Therefore, based on the position of $\vec{m}$ in one of the four OLTs, the carrier frequency is changed between $8.33 \mathrm{kHz}$ and 12.5 $\left(8.33 \times \frac{3}{2}\right) \mathrm{kHz}$. Fig. 11a and 11d show the experimental and simulated four sinusoidal line currents, $i_{a}, i_{a^{\prime}}, i_{b}$, and $i_{b^{\prime}}$; Fig. $11 \mathrm{~b}, 11 \mathrm{e}$ show the corresponding two line-line voltages, $v_{a b}$ and $v_{a^{\prime} b^{\prime}}$, respectively, of the proposed optimal technique at $M_{I}=0.577, f_{o}=50 \mathrm{~Hz}$, and output power of $3.55 \mathrm{~kW}$. The fundamental operation of ASIM in linear region can be verified from $30^{\circ}$ phase-shifted pairs, $\left\{v_{a b}, v_{a^{\prime} b^{\prime}}\right\},\left\{i_{a}, i_{a^{\prime}}\right\},\left\{i_{b}, i_{b^{\prime}}\right\}$ and $120^{\circ}$ phase-shifted pairs, $\left\{i_{a}, i_{b}\right\},\left\{i_{a^{\prime}}, i_{b^{\prime}}\right\}$. Fig. $11 \mathrm{c}$ and 11f show the frequency spectrum of experimentally obtained phase current, $i_{a}$, and line-line voltage, $v_{a b}$, respectively. The peak of the fundamental components of these current and voltage are $8.06 \mathrm{~A}$ and $300.11 \mathrm{~V}$. Although no significant harmonic is present in $v_{a b}$ spectrum, small amount of fifth harmonic $(0.17 \mathrm{~A})$ is present in phase current which arises due to machine or converter asymmetry and is unavoidable in case of ASPM running in open-loop, [18]. Fig. 11k shows the six pole voltages, $v_{x N}$, when $\vec{m}=\sqrt{3} \times 0.4 e^{j 7.5^{\circ}}$ lies in sector- 1 and the proposed hybrid technique applies OLT2. Here $x \in\left\{a, b, c, a^{\prime}, b^{\prime}, c^{\prime}\right\}$ and $N$ is DC-bus negative terminal. The switching sequence applied by $6 \phi$ inverter can be derived from this diagram, which is $\left(0,7^{\prime}\right)-\left(0,6^{\prime}\right)-\left(1,6^{\prime}\right)-$ $\left(1,1^{\prime}\right)-\left(2,1^{\prime}\right)-\left(1,1^{\prime}\right)-\left(1,6^{\prime}\right)-\left(0,6^{\prime}\right)-\left(0,7^{\prime}\right)$. This sequence matches with the sequence given in Table VI.

The analytical performance comparison of the proposed technique with existing techniques, as discussed in sectionIV, is first validated through simulation at $\gamma=2,6,10$. These are shown in Fig. 11g, 11h and 11i, respectively. The carrier frequency, $F_{s}=\frac{F_{s w}}{k_{f}}$, is varied from one technique to another based on $k_{f}$ values, as given in Table VIII. The 


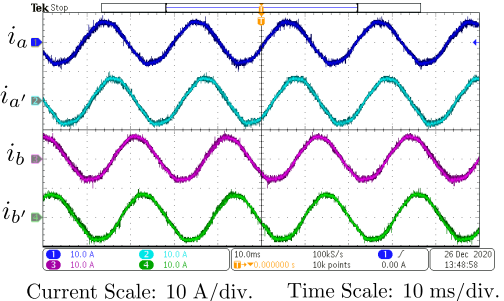

(a)Experimental $i_{a}, i_{a^{\prime}}, i_{b}, i_{b^{\prime}}$ at $M_{I}=0.577$

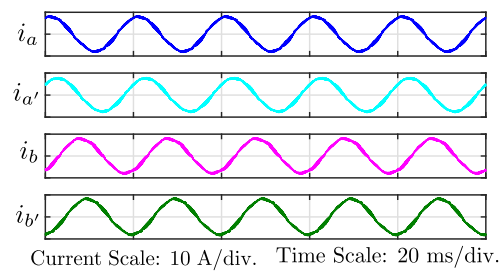

(d)Simulated $i_{a}, i_{a^{\prime}}, i_{b}, i_{b^{\prime}}$ at $M_{I}=0.577$

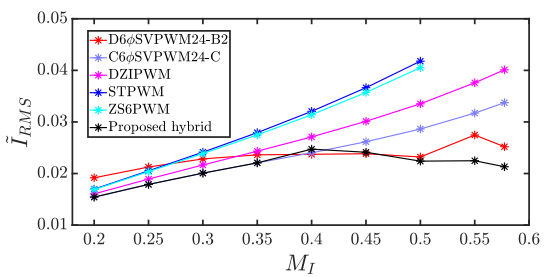

(g) Simulated $\tilde{I}_{R M S}$ vs $M_{I}$ at $\gamma=2$

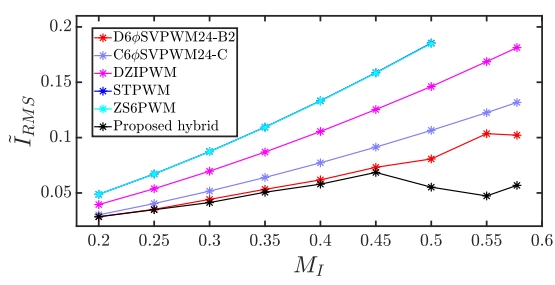

(i) Simulated $\tilde{I}_{R M S}$ vs $M_{I}$ at $\gamma=10$

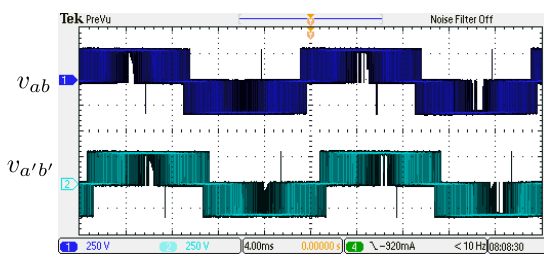

Voltage Scale: $250 \mathrm{~V} /$ div. Time Scale: $4 \mathrm{~ms} /$ div.

(b)Experimental $v_{a b}, v_{a^{\prime} b^{\prime}}$ at $M_{I}=0.577$

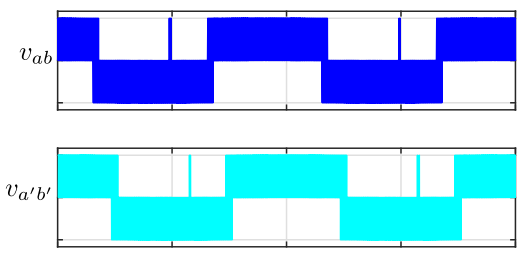

Voltage Scale: $300 \mathrm{~V} /$ div. Time Scale: $10 \mathrm{~ms} / \mathrm{div}$

(e)Simulated $v_{a b}, v_{a^{\prime} b^{\prime}}$ at $M_{I}=0.577$

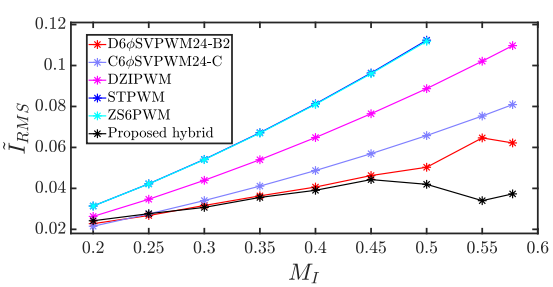

(h) Simulated $\tilde{I}_{R M S}$ vs $M_{I}$ at $\gamma=6$

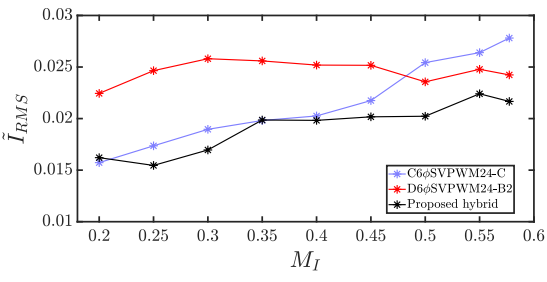

(j) Experimental $\tilde{I}_{R M S}$ vs $M_{I}$ at $\gamma=1.69$

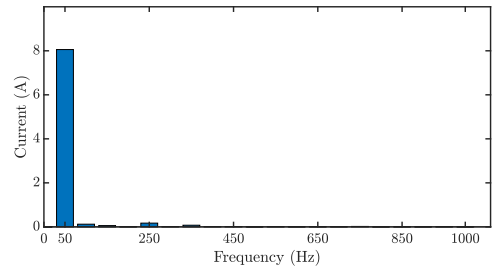

(c) Spectrum of experimental $i_{a}$ at $M_{I}=0.577$

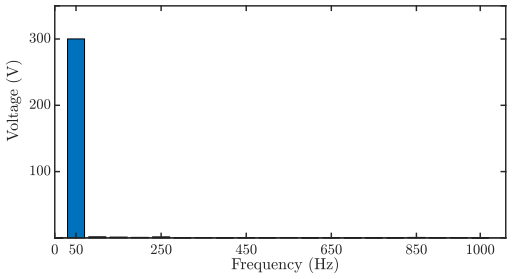

(f) Spectrum of experimental $v_{a b}$ at $M_{I}=0.577$

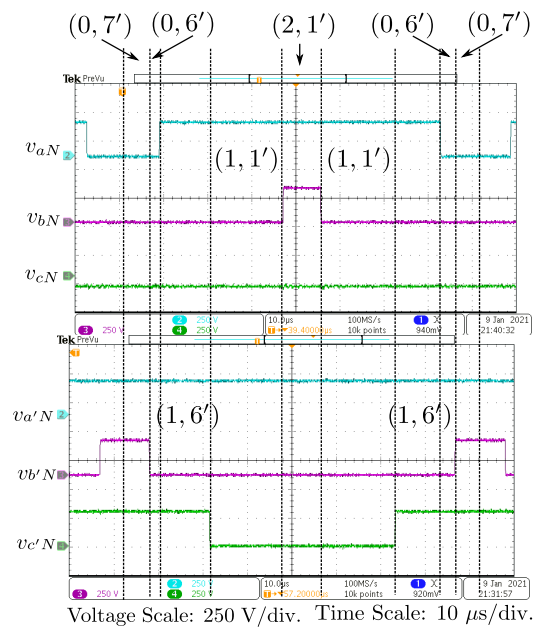

(k) Experimentally obtained sequence when OLT-2 is applied in sector-1

Fig. 11: Experimental and simulation waveforms of proposed hybrid technique and ripple-current comparison with existing techniques

simulated study also reaches to the same conclusion about the optimality of the proposed technique as discussed in section$I V$. For experimental validation of this performance comparison, we have chosen two techniques, D6 $\phi$ SVPWM24-B2 and C6 $\phi$ SVPWM24-C, whose performances are close to proposed hybrid technique for an ASPM with $\gamma=1.69$. Note, the analytical performance at $\gamma=1.69$ can be expected to be very similar to the performance at $\gamma=2$, which is given in Fig. 9a. Fig. $11 \mathrm{j}$ shows this ripple-current comparison. At lower $M_{I}$, C6 $\phi$ SVPWM24-C and proposed technique performs in the similar manner, which is better than D6 $\phi$ SVPWM24-B2. As $M_{I}$ increases, performance of D6 $\phi$ SVPWM24-B2 becomes better than $\mathrm{C} 6 \phi$ SVPWM24-C and the proposed hybrid optimal technique is superior compared to both of these techniques. $\tilde{I}_{R M S}$ for simulation and experimental results are computed by subtracting the RMS due to the fundamental and few of the low order harmonics from the total RMS current.

\section{CONCLUSION}

This paper accounts for an exhaustive set of linear modulation techniques (LMTs) of the asymmetrical six-phase machine (ASPM), which avoids a major disadvantage present in a few existing LMTs- turning on and turning off a device more than one time within a carrier period. It increases the instantaneous switching frequency of the device for a given average switching frequency. The paper first identifies the sequences per three-phase $(3 \phi)$ inverter basis, where the above limitation is overcome. After that, combining the sequences of two $3 \phi$ inverters, the paper forms eight sequence-groups. The number of possible techniques in six sequence-groups is infinite because the zero-vector dwell-times of two $3 \phi$ inverters can be distributed between the redundant zero states in infinite possible ways. This distribution impacts the current ripple of LMTs. After comparing the current-ripple performances of the techniques of all eight sequence-groups, this paper identifies five techniques, which show the optimality within the entire linear region for the whole feasible range of the machine parameter, $\gamma$. Only one of these five techniques exists in the literature. After that, an optimal hybrid strategy is proposed which applies the best of these five techniques in each carrier cycle depending upon the position of the reference voltage vector. For any given $\gamma$ within the feasible range, the proposed hybrid optimal strategy shows the best ripple current performance in the entire range of modulation indices compared with the existing best-performing techniques, which don't have the 
disadvantage mentioned above. The proposed method shows significant improvement in the ripple performance at higher values of $\gamma$ and modulation indices. The theoretical analysis is verified through simulation and experiment on an ASPM with $\gamma=1.69$ up to $3.5 \mathrm{~kW}$ power level.

\section{REFERENCES}

[1] F. Barrero and M. J. Duran, "Recent advances in the design, modeling, and control of multiphase machines-part i," IEEE Transactions on Industrial Electronics, vol. 63, no. 1, pp. 449-458, 2015.

[2] E. Levi, "Advances in converter control and innovative exploitation of additional degrees of freedom for multiphase machines," IEEE Transactions on Industrial Electronics, vol. 63, no. 1, pp. 433-448, 2015.

[3] A. S. Abdel-Khalik, A. M. Massoud, and S. Ahmed, "Effect of dc-link voltage limitation on postfault steady-state performance of asymmetrical six-phase induction machines," IEEE Transactions on Industrial Electronics, vol. 65, no. 9, pp. 6890-6900, 2018.

[4] A. Cervone, M. Slunjski, E. Levi, and G. Brando, "Optimal thirdharmonic current injection for asymmetrical multiphase pmsms," IEEE Transactions on Industrial Electronics, 2020.

[5] Y. Zhao and T. A. Lipo, "Space vector pwm control of dual threephase induction machine using vector space decomposition," IEEE Transactions on industry applications, vol. 31, no. 5, pp. 1100-1109, 1995.

[6] D. Hadiouche, L. Baghli, and A. Rezzoug, "Space-vector pwm techniques for dual three-phase ac machine: analysis, performance evaluation, and dsp implementation," IEEE Transactions on Industry Applications, vol. 42, no. 4, pp. 1112-1122, 2006.

[7] K. Marouani, L. Baghli, D. Hadiouche, A. Kheloui, and A. Rezzoug, "A new pwm strategy based on a 24-sector vector space decomposition for a six-phase vsi-fed dual stator induction motor," IEEE Transactions on Industrial Electronics, vol. 55, no. 5, pp. 1910-1920, 2008.

[8] C. Wang, K. Wang, and X. You, "Research on synchronized svpwm strategies under low switching frequency for six-phase vsi-fed asymmetrical dual stator induction machine," IEEE Transactions on Industrial Electronics, vol. 63, no. 11, pp. 6767-6776, 2016.

[9] A. M. Hava, R. J. Kerkman, and T. A. Lipo, "Simple analytical and graphical methods for carrier-based pwm-vsi drives," IEEE transactions on power electronics, vol. 14, no. 1, pp. 49-61, 1999.

[10] A. Bakhshai, G. Joos, and H. Jin, "Space vector pwm control of a splitphase induction machine using the vector classification technique," in Applied Power Electronics Conference and Exposition, 1998. APEC'98. Conference Proceedings 1998., Thirteenth Annual, vol. 2, pp. 802-808. IEEE, 1998.

[11] R. Bojoi, A. Tenconi, F. Profumo, G. Griva, and D. Martinello, "Complete analysis and comparative study of digital modulation techniques for dual three-phase ac motor drives," in Power Electronics Specialists Conference, 2002. pesc 02. 2002 IEEE 33rd Annual, vol. 2, pp. 851-857. IEEE, 2002.

[12] S. Paul and K. Basu, "A three-phase inverter based overmodulation strategy of asymmetrical six-phase induction machine," IEEE Transactions on Power Electronics, 2020.

[13] P. R. Rakesh and G. Narayanan, "Analysis of sine-triangle and zerosequence injection modulation schemes for split-phase induction motor drive," IET Power Electronics, vol. 9, no. 2, pp. 344-355, 2016.

[14] J. Prieto, E. Levi, F. Barrero, and S. Toral, "Output current ripple analysis for asymmetrical six-phase drives using double zero-sequence injection pwm," pp. 3692-3697, 2011.

[15] V. Blasko, "Analysis of a hybrid pwm based on modified spacevector and triangle-comparison methods," IEEE Transactions on industry applications, vol. 33, no. 3, pp. 756-764, 1997.

[16] D. G. Holmes, "The significance of zero space vector placement for carrier-based pwm schemes," IEEE Transactions on Industry Applications, vol. 32, no. 5, pp. 1122-1129, 1996.

[17] D. Hadiouche, H. Razik, and A. Rezzoug, "On the modeling and design of dual-stator windings to minimize circulating harmonic currents for vsi fed ac machines," IEEE Transactions on industry applications, vol. 40, no. 2, pp. 506-515, 2004.

[18] H. S. Che, E. Levi, M. Jones, W.-P. Hew, and N. A. Rahim, "Current control methods for an asymmetrical six-phase induction motor drive," IEEE Transactions on Power Electronics, vol. 29, no. 1, pp. 407-417, 2013. 